\title{
A CHARACTER FORMULA FOR THE DISCRETE SERIES OF A SEMISIMPLE LIE GROUP
}

\author{
BY JORGE VARGAS
}

\begin{abstract}
For a semisimple Lie group $G$, we provide an explicit formula for the discrete series characters $\theta_{\lambda}$ restricted to the identity component of a split Cartan subgroup, whenever the parameter lies in a so-called Borel-de Siebenthal chamber and $G$ has both a compact Cartan subgroup and a split Cartan subgroup.
\end{abstract}

Let $G$ be a connected semisimple Lie group with finite center. The discrete series of $G$ is, by definition, the set of equivalence classes of irreducible unitary representations $\pi$, such that $\pi$ occurs discretely in the left (or right) regular representation of $G$. According to Harish-Chandra [3], $G$ has a nonempty discrete series if and only if $G$ contains a compact Cartan subgroup. Thus we fix a compact Cartan subgroup $B \subset G$, and a maximal compact subgroup $K \subset G$ which contains $B$. Let $\mathfrak{g}, \mathfrak{f}, \mathfrak{b}$ be the Lie algebras of $G, K, B$, and $\mathfrak{g}^{\mathbf{C}}, \mathfrak{f} \mathbf{C}, \mathfrak{b} \mathbf{C}$ their complexifications. Let $\Phi=\Phi\left(\mathfrak{g}^{\mathrm{C}}, \mathfrak{b}^{\mathrm{C}}\right)$ be the root system of $\left(\mathfrak{g}^{\mathrm{C}}, \mathfrak{b}^{\mathrm{C}}\right)$. A root $\alpha \in \Phi$ is called compact (respectively noncompact) if its root space lies in $\mathfrak{f}^{\mathbf{C}}$ (respectively the orthogonal complement of $\mathfrak{f}^{\mathbf{C}}$ ). The differentials of the characters of $B$ form a lattice $\Lambda \subset i \mathfrak{b}^{*}\left(\mathfrak{b}^{*}=\right.$ dual space of $\left.\mathfrak{b}\right)$. The killing form induces a positive definite inner product (, ) on $i \mathfrak{b} *$. An element $\lambda \in \Lambda$ is called nonsingular if $(\lambda, \alpha) \neq 0$ for every $\alpha \in \Phi$. We set $W=W(G, B)=$ Weyl group of $B$ in $G$. Equivalently, $W$ can be described as the group generated by the reflection about the compact roots in $i \mathfrak{b}^{*}$.

In order to state Harish-Chandra's enumeration of the discrete series [3], we assume, without loss of generality, that $G$ is acceptable in the sense of HarishChandra. Then, for each nonsingular $\lambda \in \Lambda$, there exists exactly one tempered ${ }^{1}$ invariant eigendistribution $\theta_{\lambda}$ on $G$, such that

$$
\left.\theta_{\lambda}\right|_{B \cap G^{\prime}}=(-1)^{q} \frac{\Sigma_{w \in W} \operatorname{sgn} w e^{w \lambda}}{\Pi_{\alpha \in \Phi,(\alpha, \lambda)>0}\left(e^{\alpha / 2}-e^{-\alpha / 2}\right)} .
$$

Here $q=1 / 2 \operatorname{dim} G / K$, and $G^{\prime}=$ set of regular semisimple points in $G$. Every $\theta_{\lambda}$ is the character of a discrete series representation, and conversely. Moreover, $\theta_{\lambda}$ $=\theta_{\mu}$ if and only if $\lambda$ belongs to the $W$-orbit of $\mu$.

Summary of doctoral dissertation, submitted to the Department of Mathematics at Columbia University in 1977; received by the editors October 2, 1979.

AMS (MOS) subiect classifications (1970). Primary $22 \mathrm{E} 45$.

Key words and phrases. Semisimple Lie groups, representations, discrete series, character formulas.

${ }^{1}$ A distribution $\theta$ on $G$ is tempered if it extends to the Schwartz space of rapidly decreasing functions [3]. 
When $G / K$ is a hermitian symmetric space, there exist positive root systems in $\Phi$ such that the sum of two noncompact positive roots is never a root. If $\lambda$ is dominant with respect to such a positive root system, $\theta_{\lambda}$ is the character of one of the so-called holomorphic discrete series representations. In this special situation, S. Martens [5] and H. Hecht [4] have given explicit global formulas for the characters $\theta_{\lambda}$. Whether or not $G / K$ is hermitian symmetric, there exist positive root systems which satisfy the following condition [1]:

for each noncompact simple factor $G_{i}$ of $G$, there exists exactly one noncompact simple root $\beta_{i}$, and this root $\beta_{i}$ occurs at most twice in the highest root of $G_{i}$.

(Borel-de Siebenthal property). The problem of computing the discrete series characters $\theta_{\lambda}$ globally can be reduced, at least in principle, to the following rather special situation:

(a) $G$ is simple and has both a compact Cartan subgroup $B$ and a split Cartan subgroup $A$.

(b) Compute $\theta_{\lambda}$ restricted to the identity component of a split Cartan subgroup $A$.

(c) The system of positive roots $\Psi=\{\alpha \in \Phi \mid(\alpha, \lambda)>0\}$ has the Borelde Siebenthal property. (See Schmid [6].)

From now on, let $G, \lambda, \Psi, A$ be as in (a)-(c), and $d$ an inner automorphism of $\mathfrak{g}^{\mathbf{C}}$ such that $d: \boldsymbol{b} \mathbf{C} \stackrel{\sim}{\longrightarrow} \mathbf{a}$.

We denote the identity component of $A$ by $A^{\circ}$ and define

$$
C=\left\{\exp X \mid X \in \mathfrak{a},\left\langle\alpha, d^{-1} X\right\rangle\langle 0 \text { for all } \alpha \in \Psi\} .\right.
$$

The closure of $C$ and its conjugates cover $A^{\circ}$. Our main result provides an explicit formula for the restrictions of $\theta_{\lambda}$ to $A^{\circ}$. This formula involves a particular element $t$ of the Weyl group of $\left(g^{\mathbf{C}}, \mathfrak{b}^{\mathbf{C}}\right)$, whose description we defer until later.

THEOREM. Let $W_{U}$ be the subgroup of $W$ generated by the compact simple roots for $\Psi$. Then

$$
\left.\theta_{\lambda}\right|_{C}=(-1)^{q} \frac{|W|}{\left|W_{U}\right|} \frac{\Sigma_{w \in W} \operatorname{sgn}(t w) e^{t w \lambda}}{\Pi_{\alpha \in \Psi}\left(e^{\alpha / 2}-e^{-\alpha / 2}\right)} \circ d^{-1}
$$

Let $\beta$ be the noncompact simple root for $\Psi$. Then $\beta$ is as long as or longer than any noncompact root, and the system $\Phi^{\prime}$ of the roots in $\Phi$ orthogonal to $\beta$ has at most three irreducible components. Moreover if $\Phi^{\prime}$ has more than one connected component, then all but perhaps one are of type $A_{1}$, and all the $A_{1}$ type components consist of noncompact roots. Two roots $\alpha_{1}, \alpha_{2} \in \Phi$ are said to be strongly orthogonal if $\alpha_{1} \pm \alpha_{2} \notin \Phi$. For any strongly orthogonal subset $S \subset \Phi$ consisting of noncompact roots, we set $\Phi_{S}=$ Q-linear span of $S$ in $\Phi$. 
Lemma 1. Each irreducible component of $\Psi \cap \Phi_{S}$ has the Borel-de Siebenthal property.

We now define a family of sub-root systems $\Phi=\Phi^{0}, \Phi^{1}, \ldots, \Phi^{m}$ inductively, as follows: $\Phi^{i+1}$ is the set of roots in $\Phi^{i}$ orthogonal to the noncompact simple roots for $\Psi \cap \Phi^{i}$, until the process stops. Let $S_{0}$ be the set consisting of all positive noncompact roots that are simple roots for some $\Psi \cap \Phi^{i}, 0 \leqslant i \leqslant m$.

Lemma 2. (a) $S_{0}$ is a strongly orthogonal set which spans $\Phi$ over $\mathbf{Q}$.

(b) $S_{0}$ contains at most one short root.

The next proposition describes the element $t$ of the Weyl group of $\left(g^{C}, \mathfrak{b}^{C}\right)$ which was used in the statement of the main result.

Proposition. There exists a unique tin the Weyl group of $\left(3^{\mathrm{C}}, 6^{\mathbf{C}}\right)$ such that

(1) $t$ is a product of reflections about roots in $S_{0}$.

(2) If $\beta$ occurs twice in the highest root, then $t \neq 1$.

(3) $t$ takes any long simple root into a noncompact root.

(4) $(t w \lambda, \mu) \geqslant 0$ for every $w \in W_{U}$ and $\lambda, \mu$ dominant integral with respect to $\Psi$.

From the Proposition, one can deduce the following properties of $t$ :

(a) If $\alpha_{1}, \alpha_{2}$ are two adjacent long simple roots, then $\operatorname{sgn} t \alpha_{1} \neq \operatorname{sgn} t \alpha_{2}$.

(b) Assume $\beta$ occurs twice in the highest root. Then $t$ fixes any short root in $S_{0}$.

(c) If $S_{0}$ does not contain short roots, $t \alpha=\alpha$ for any short simple root $\alpha$.

(d) Again under the assumption that $\beta$ occurs twice in the highest root, if $S_{0}$ does contain short roots, $t \alpha$ is noncompact for any short simple root $\alpha$. The proof of the theorem procedes by induction on the dimension of $G$. The crux of the matter is to verify the consistency of our formula with Harish-Chandra's matching conditions [2]. Details will appear elsewhere.

Finally, I would like to express my deep gratitude to Professor Wilfried Schmid for his advice and interest.

\section{REFERENCES}

1. A. Borel, et J. de Siebenthal, Les sous-groupes fermés de rang maximum des groupes de Lie clos, Comm. Math. Helv. 23 (1949), 200-221.

2. Harish-Chandra, Invariant eigendistributions on a semi-simple Lie group, Trans. Amer. Math. Soc. 119 (1965), 457-508.

3. Discrete series for semi-simple Lie groups. II, Acta Math. 116 (1966), 1-111. $213-226$

4. H. Hecht, The characters of Harish-Chandra representations, Math. Ann. 219 (1976),

5. S. Martens, The characters of the holomorphic discrete series, Proc. Nat. Acad. Sci. U.S.A. vol. 72, no. 9, 1975, pp. 3275-3276.

6. W. Schmid, On the characters of the discrete series, Invent. Math. 30 (1975), 47-144.

DEPARTMENT OF MATHEMATICS, NATIONAL UNIVERSITY OF CORDOBA, CORDOBA, ARGENTINA 